\title{
EL CONSTITUCIONALISMO VIVIENTE: MÉTODO INTERPRETATIVO, PRESUPUESTOS SEMÁNTICOS Y DIFICULTADES
}

LUCIANO D. LAISE 
SUMARIO

PLANTEAMIENTO: EL DESAFÍO DE SISTEMATIZAR LA NATURALEZA Y PRESUPUESTOS SEMÁNTICOS DE LAS METODOLOGÍAS INTERPRETATIVAS VIVIENTES. I. PRIMERA DIRECTIVA INTERPRETATIVA: IDENTIFICAR LAS TENDENCIAS JURISPRUDENCIALES Y DOCTRINARIAS. II. SEGUNDA DIRECTIVA INTERPRETATIVA: RECONOCER A LOS ACTORES CONSTITUCIONALES ENCARGADOS DE MATERIALIZAR LAS ASPIRACIONES INCORPORADAS EN LA CONSTITUCIÓN. III. TERCERA DIRECTIVA INTERPRETATIVA: RECONOCER LA ESTRUCTURA Y FUNCIONAMIENTO MATERIAL DEL GOBIERNO DEL ESTADO. IV. CUARTA DIRECTIVA INTERPRETATIVA: PROYECTAR LAS CONSECUENCIAS (POLÍTICAS) DE LA DECISIÓN JUDICIAL SOBRE LA ESTRUCTURA SOCIAL V. QUINTA DIRECTIVA INTERPRETATIVA: ASUMIR LA INVENCIBILIDAD DEL TEXTO CONSTITUCIONAL Y LA TRADICIÓN FRENTE A LAS ASPIRACIONES, IDEALES O VALORES ACTUALES. VI. LOS PRESUPUESTOS SEMÁNTICOS DE UNA INTERPRETACIÓN EVOLUTIVA DE LOS DERECHOS: UNA TEORÍA DEL SIGNIFICADO DE NATURALEZA CONVENCIONALISTA. VII. A MODO DE CONCLUSIÓN. 


\title{
EL CONSTITUCIONALISMO VIVIENTE: MÉTODO INTERPRETATIVO, PRESUPUESTOS SEMÁNTICOS Y DIFICULTADES*
}

\author{
LUCIANO D. LAISE**
}

\author{
PLANTEAMIENTO: EL DESAFÍO DE SISTEMATIZAR LA \\ NATURALEZA Y PRESUPUESTOS SEMÁNTICOS DE LAS \\ METODOLOGÍAS INTERPRETATIVAS VIVIENTES
}

En el presente trabajo se reconstruirá la metodología y los presupuestos semánticos de la interpretación viviente o evolutiva de los derechos fundamentales. Se trata de un objetivo que resulta especialmente desafiante porque, en general, ni la teoría ni la práctica interpretativa suelen sistematizar las directivas de esta metodología evolutiva ${ }^{1}$. Esto pareciera corroborar una generalización que Michael S. Moore ha sugerido hace ya un tiempo: los promotores de una teoría de la interpretación de los derechos se limitan a defender una determinada metodología sobre la base de argumentos que descansan en alguna noción de autoridad, sin prestar una cuidadosa

* Este trabajo se inserta en el proyecto de investigación titulado: «Coordinación y autoridad en el Estado Constitucional de Derecho. Desafíos teóricos y prácticos en un contexto de fragmentación moral» (Código PICTO-2016-0095), financiado por la Agencia Nacional de Promoción Científica y Tecnológica (Argentina).

** Doctor en Derecho (U. Austral, Argentina). Investigador asistente de la Carrera de Investigador Científico del CONICET, lugar de trabajo: Departamento de Ciencias Sociales, Jurídicas y Económicas de la Universidad Nacional de Chilecito, 9 de Julio 22, Chilecito, La Rioja, Argentina. ORCID N. ${ }^{\circ}$ : https://orcid.org/0000-0003-4249-5948. E-Mail: lucianolaise@conicet.gov.ar

1 Kavanagh, Aileen, "The Idea of a Living Constitution», Canadian Journal of Law and Jurisprudence, vol. 16, 2003, p. 55. También se han identificado algunas pocas contribuciones a la sistematización de constitucionalismo viviente en el mundo hispanoparlante, por ejemplo, Candia Falcón, Gonzalo, «Ponderación y constitución viviente como expresión del mismo fenómeno constitucional: la experiencia norteamericana», Revista Española de Derecho Constitucional, vol. 107, 2016, pp. 41-74. https://doi. org/10.18042/cepc/redc.107.02 
atención a su caracterización metodológica ${ }^{2}$. En el caso específico de la interpretación evolutiva, según sus críticos, esto se debería a que esta metodología resultar ilegitima porque implica emitir un cheque en blanco a favor de los jueces constitucionales, como bien lo indica Strauss ${ }^{3}$.

La labor reconstructiva que supone esta contribución se focalizará en la identificación y sistematización de las directivas interpretativas que caracteriza a las metodologías bajo estudio. En tal sentido resulta preciso distinguir entre dos versiones de las metodologías evolutivas ${ }^{4}$. Por un lado, una interpretación evolutiva complementaria o suplementaria que defiende la posibilidad $-\mathrm{y}$, en ocasiones, la necesidad- de aggiornar el texto constitucional a los tiempos actuales ${ }^{5} \mathrm{y}$, por el otro, una versión alternativa que supera la mera complementación y defiende la deseabilidad de sustituir el texto constitucional por las aspiraciones o valores morales contemporáneos.

La metodología interpretativa evolutiva sustitutiva ha sido fuertemente cuestionada porque implica reemplazar el texto constitucional por una serie de factores ajenos a él tales como aspiraciones sociales o morales, precedentes judiciales o una remisión a un consenso social. Meese ha llegado a plantear que se sacrifica a la democracia si se adopta una interpretación evolutiva de los derechos fundamentales, porque

2 Moore, Michael S., «Justifying the Natural Law Theory of Constitutional Interpretation», Fordham Law Review, vol. 69, 2000, p. 2098. En un sentido similar, Suárez Rodríguez, José Julián, «La teoría de los principios y el estado constitucional. Luces y sombras», en Retos del derecho constitucional contemporáneo, ed. Miguel et alii Ayuso, Bogotá, Astrea-Editorial Universidad de La Sabana, 2013, pp. 161-177. Bradley Miller plantea que se ha desarrollado abundantemente la deseabilidad y necesidad de la interpretación evolutiva de la Constitución. Sin embargo, el citado autor sostiene que se ha teorizado escasamente sobre la metodología interpretativa que supone el constitucionalismo viviente. Miller, Bradley W., «Beguiled By Metaphors: The Living Tree and Originalist Constitutional Interpretation in Canada», Canadian Journal of Law and Jurisprudence, vol. 22, n. ${ }^{\circ}$, 2009 , p. 333

3 Strauss, David A., The Living Constitution, New York, Oxford University Press, 2010, p. 118. «Because judicial power resides in the authority to give meaning to the Constitution, the debate is really a debate about how to read the text, on what is legitimate interpretation». Brennan, William J., «The Constitution of the United States: Contemporary Ratification», South Texas Law Review, vol. 27 , n. 3,1985 , p. 435.

4 En contra de una teoría de la interpretación constitucional que suponga una la distinción entre suplantación y una complementación de las disposiciones constitucionales a partir de los valores morales y políticos contemporáneos, Dworkin, Ronald, Freedom's Law: The Moral Reading of the American Constitution, Cambridge-EE.UU., Harvard University Press, 1996, 4. Para una categorización similar en el ámbito hispanoparlante, Risso Ferrand, Martín José, «Mutación e interpretación evolutiva de la constitución: dos casos uruguayos», Estudios Constitucionales: Revista del Centro de Estudios Constitucionales, vol. 15, n. ${ }^{\circ}$ 1, 2017, p. 218.

5 Entre los partidarios de este enfoque metodológico, Grey, Thomas C., «Do We Have an Unwritten Constitution?», Stanford Law Review, vol. 27, n. ${ }^{\circ}$ 3, 1974, p. 709. Kavanagh, "The Idea of a Living Constitution», p. 87. Lessig, Lawrence, «Understanding Changed Readings: Fidelity and Theory», Stanford Law Review, vol. 47, n. ${ }^{\circ}$ 3, 1994, pp. 396-397. Waluchow, Will, «Constitutions as Living Trees: An Idiot Defends», Canadian Journal of Law and Jurisprudence, vol. 18, n. ${ }^{\circ}$ 2, 2005, p. 222. 
en este caso serían los jueces quienes deciden si se aplica o no la Constitución ${ }^{6}$. Dejando de lado a la afirmación radical de Meese —uno de los principales epígonos del originalismo intencionalista ${ }^{7}$ - este autor pone de relieve un punto que es importante subrayar: las razones que permiten evaluar estas metodologías interpretativas han de apreciarse en función de argumentos normativos y no tanto a través de una necesidad de tipo conceptual-analítica que se sigue del concepto de constitución o tratado de derechos humanos. De hecho, en este trabajo no se afirmará la futilidad de esta última aproximación metodológica, pero se emprenderá un camino sustancialmente diferente ${ }^{8}$.

Más específicamente, en las páginas que siguen se sostendrá la idea de que es deseable tratar al texto constitucional como la ley suprema de un Estado, no pudiendo este ser derrotado por los valores o aspiraciones sociales que recogiese la práctica constitucional actual ${ }^{9}$. En efecto, la tesis normativa que se defenderá se justifica en el entendimiento de que la interpretación evolutiva sustitutiva se encuentra en tensión con un principio recogido en el texto constitucional que el control de constitucionalidad pretende implementar: la supremacía de la Constitución ${ }^{10}$.

Sin embargo, también es cierto que la metodología interpretativa evolutiva de tipo complementaria supone otras dificultades que no dependen principalmente de su grado de compatibilidad con el principio de supremacía constitucional. Se trata, en efecto, de cuestiones problemáticas que se siguen de las bases o presupuestos semánticos de las metodologías evolutivas. Así, en este trabajo se argumentará que una interpretación evolutiva de tipo complementaria podrá resultar legítima, pero en la medida en que tal metodología no se apoye en una semántica convencionalista. Esta teoría del significado afirma, siguiendo el camino trazado por Austin hace ya un tiempo, que la extensión —o referencia- de los conceptos depende principalmente de construcciones sociales de significado o, dicho en los términos de la filosofía del

${ }^{6}$ Meese III, Edwin, «The Supreme Court of the United States: Bulwark of a Limited Constitution», South Texas Law Review, vol. 27, n. ${ }^{\circ} 3,1985$, p. 465.

7 Laise, Luciano D., «La interpretación de la Constitución de Estados Unidos según el originalismo intencionalista: método interpretativo, presupuestos semánticos y dificultades», Historia Constitucional, vol. 18, 2017, pp. 245-292.

${ }^{8}$ Finnis sugiere que la elaboración de una teoría del Derecho basada en la canónica distinción entre una metodología «analítica» o «descriptiva» y «crítica» — también llamada «normativa»— no resulta satisfactoria para brindar una descripción completa de la realidad jurídica. Esto se debe a que la creación y el mantenimiento de un sistema jurídico descansa en «razones para la acción» y esto presupone y/o propone juicios evaluativos de las conductas humanas. Por ello, toda descricpión del sistema jurídico precisa identificar aquellas valoraciones que modelan a ese sistema. Finnis, John, Collected Essays: Philosophy of Law, vol. 4, New York, Oxford University Press, 2011, p. 165.

9 Moore, Michael S., «Do We Have an Unwritten Constitution?», Southern California Law Review, vol. 63, n. ${ }^{\circ} 1,1989$, p. 119. «Activist judicial revie that proceeds without taking account of the popular view of the importance of writings lacks the consent of the governed». Grey, Thomas C., «The Uses of an Unwritten Constitution», Chicago-Kent Law Review, vol. 64, n. ${ }^{\circ}$ 1, 1988, p. 231.

${ }^{10}$ Barnett, Randy E., «The Original Meaning of the Judicial Power», Supreme Court Economic Review, vol. 12, 2004, p. 137. 
lenguaje actual, «convenciones lingüísticas» ${ }^{11}$. En efecto, resulta preciso aclarar que para una semántica convencionalista no necesariamente el significado de los conceptos es construido entera o completamente por medio consensos o convenciones. El punto que defiende esa teoría semántica es que el elemento convencional prevalece sobre la referencia de los conceptos.

El abordaje de los problemas teóricos a estudiar en este artículo se basará primordial pero no exclusivamente en doctrina y jurisprudencia norteamericana — tanto de Estados Unidos de América como de Canadá-. La razón que justifica tal elección metodológica obedece a los profusos y extensos debates sobre interpretación viviente o evolutiva que se dieron en Canadá. De hecho, fue la jurisprudencia constitucional canadiense la que acuñó la metáfora de la Constitución como un «árbol viviente capaz de crecer y expandirse dentro de sus límites naturales» ${ }^{12}$. La selección del caso estadounidense responde a dos razones. En primer lugar, como bien advierte Carbonell, cuanto más difícil es seguir el camino de la reforma, enmienda o cambio formal de una constitución, mayor es el grado de desarrollo teórico de sus métodos interpretativos $^{13}$. Así, puesto que la constitución estadounidense es la más antigua de aquellas que actualmente se encuentran vigentes, es sencillo apreciar que en tal sistema constitucional muchos de los cambios constitucionales se han canalizado por medio de prácticas interpretativas — principalmente de la Corte Suprema de Justicia— ${ }^{14}$. Estos cambios, en efecto, solo podrían ser explicados apelando a una metodología viviente o evolutiva de la interpretación constitucional ${ }^{15}$.

El orden formal que se adoptará en esta investigación será el siguiente: i) reconstruir y describir sistemáticamente a las directivas interpretativas de las metodologías evolutivas —epígrafes 1 a 6-. Luego, (ii) se pondrá el foco en la evaluación crítica de estas metodologías de interpretación de los derechos —epígrafe 7—. De esta manera se evitará incurrir en una «falacia del hombre de paja» ${ }^{16}$; esto es, una carac-

11 Austin, J. L., «How to Talk. Some Simple Ways», Proceedings of the Aristotelian Society, vol. 53, 1953, p. 229. Para un trabajo que defiende la idea de que la regla de reconocimiento del Derecho depende de una convención social, Ortega García, Ramón, «The Conventionality of Law», Problema: Anuario de Teoría y Filosofía del Derecho, vol. 10, 2016, p. 246. DOI: http://dx.doi.org/10.22201/ iij.24487937e.2016.10.8201. En contra, señalando las dificultades de una teoría de la interpretación jurídica basada en una semántica convencionalista, Laise, Luciano D., «Convencionalismo semántico e interpretación jurídica», Problema: Anuario de Teoría y Filosofía del Derecho, vol. 11, 2017, pp. 274-339.

12 «Edwards v. Attorney General of Canada» 1930, A.C. 124, 136 British Privy Council. [En adelante: «Edwards»].

13 Carbonell Sánchez, Miguel, «Sobre la Constitución Viviente», Estudios Constitucionales: Revista del Centro de Estudios Constitucionales, vol. 10, n. ${ }^{\circ}$ 2, 2012, p. 609.

${ }^{14}$ Strauss, David A., «Do We Have a Living Constitution?», Drake Law Review, vol. 59, n. ${ }^{4}$, 2011, p. 976.

15 Ibid., p. 976.

${ }_{16}$ Una falacia de hombre de paja supone «Misrepresenting an opponent's position or argument, usually for the purpose of making it easier to attack», Damer, T. Edward, Attacking Faulty Reasoning: A Practical Guide to Fallacy-Free Arguments, 6 ed., Belmont, Wadsworth, 2008, p. 204. En un sentido similar, García Damborenea, Ricardo, Diccionario de Falacias, Madrid, Biblioteca Nueva, 2000, p. 60. 
terización simplista de la interpretación evolutiva de los derechos que solo recoge los aspectos más débiles o inconsistentes de tales metodologías para discutirlas con suma facilidad. Por el contrario, se procurará remarcar tantos los aciertos como las limitaciones y desafíos del tema bajo estudio en la presente investigación. Por último, (iii) se recapitularán las principales contribuciones a las que se arribará en este trabajo.

\section{PRIMERA DIRECTIVA INTERPRETATIVA: IDENTIFICAR LAS TENDENCIAS JURISPRUDENCIALES Y DOCTRINARIAS}

La interpretación evolutiva de los derechos exige más de un análisis de tendencias que de principios estáticos ${ }^{17}$. Esto supone que las decisiones constitucionales han de ser optimizadas por medio del estudio de convergencias históricas sobre las explicaciones doctrinarias acerca de lo que significan las disposiciones constitucionales, junto con la evaluación crítica del contexto social en el que el Derecho se desarrolla y evoluciona ${ }^{18}$. Así, el texto constitucional no sería un documento estático sino, como lo ilustra la jurisprudencia canadiense, un «árbol viviente capaz de crecer y expandirse dentro de sus límites naturales» ${ }^{19}$. Esto se debe a que el lenguaje de la Constitución, «no es un cristal transparente e inmutable, sino la piel de un pensamiento viviente que puede variar significativamente de color y contenido, de acuerdo con las circunstancias y la época en que es utilizado», tal como plantea Holmes ${ }^{20}$.

De esta manera, la Constitución terminaría siendo un instrumento que se desarrolla y adapta a las nuevas circunstancias contemporáneas y a la mutación de las creencias normativas, siempre dentro de los contornos propios de su función institucional; esto es, limitar el campo de acción del gobierno y de los particulares ${ }^{21}$. Ahora bien, ¿cómo se detectan esas tendencias doctrinarias y jurisprudenciales? Pues, en primer lugar, observando detenidamente la jurisprudencia de los órganos encargados de interpretar los derechos fundamentales. Esto supone identificar un punto de partida en el pasado, una delimitación de lo que ha venido ocurriendo en el presente, para así elaborar una extrapolación de lo que sucederá en el futuro ${ }^{22}$.

${ }_{17}$ Miller, Arthur S., «Change and the Constitution», Law and the Social Orderg, vol. 1, 1970, p. 235. Miller, Arthur S., «Notes on the Concept of the Living Constitution», George Washington Law Review, vol. 31, 1962, p. 890.

18 Miller, «Change and the Constitution», p. 236.

19 «dwards», p. 136.

20 «Towne v. Eisner, Collector of United States Internal Revenue for the Third District of the State of New York», 245 U.S. 418, 425 voto en disidencia del justice Holmes [la traducción es del autor de este trabajo]. En un sentido similar, Brennan, «The Constitution of the United States: Contemporary Ratification», p. 438.

21 Waluchow, Will, A Common Law Theory of Judicial Review: The Living Tree, New York, Cambridge University Press, 2007, p. 884.

22 Miller, «Notes on the Concept of the Living Constitution», p. 891. Miller, «Change and the Constitution», p. 237. 
Estas tendencias constitucionales no son sino las expectativas depositadas en un futuro que se construye a partir de una herencia legada por el pasado. Esto implica un método de historiografía constitucional, basada en un concepto reflexivo de «historia», que pretende explicar, fundamentar y legitimar los resultados interpretativos a partir de una conexión entre el futuro y el pasado ${ }^{23}$. Estas tendencias históricas se conocen, pues, a través de la intermediación de la historia constitucional que vincula experiencias concretas y expectativas particulares ${ }^{24}$. Dicho de otra manera, casi parafraseando a Holmes, conocer la extensión de las disposiciones constitucionales supone un estudio meticuloso de la historia ${ }^{25}$.

Miller profundiza lo dicho anteriormente y subraya que este análisis de tendencias ha de realizarse en dos niveles que se interrelacionan recíprocamente: 1) la evolución particular de algunas disposiciones constitucionales y 2) la organización del gobierno en sí misma considerada ${ }^{26}$. Así, este examen de las tendencias hace referencia, por un lado, a una serie de derechos de los individuos frente a los excesos en que pudiera incurrir el ejercicio del gobierno del Estado y, por el otro, designa un proceso de igualamiento de los individuos entre $\mathrm{si}^{27}$. Es menester remarcar la conexión entre la evolución de los derechos de los individuos y la visión del gobierno de la hora presente, ya que de lo contrario se podría arribar a resultados paradójicos o contradictorios, como bien advierte Miller.

Siguiendo tal orden de ideas, por ejemplo, la tendencia actual parece ser partidaria de un gobierno que interviene cada vez más en la sociedad y en la economía para posibilitar que un mayor número de personas goce de mayores niveles de autonomía personal $^{28}$. La expansión de la regulación estatal se explica a partir de la pretensión de un elevado nivel de satisfacción de un elenco de derechos individuales. De hecho, pareciera ser un lugar común que aumenta el catálogo de derechos individuales a la par que aumenta la intervención del gobierno para asegurar tales derechos ${ }^{29}$. En efecto, la proyección que Miller formuló hace ya un buen tiempo (1982), con relación a que no solo se expandirá, sino que se acelerará la concentración de poder en el órgano ejecutivo del gobierno, no ha perdido una pizca de actualidad ${ }^{30}$.

${ }^{23}$ Ibid., p. 237. Koselleck, Reinhart, Historia-Historia, trad. Antonio Gómez Ramos, 2da ed., Madrid, Trotta, 2010, p. 109.

${ }^{24}$ Koselleck, Reinhart, Futures Past: On the Semantics of Historical Time, trad. Keith Tribe, New York, Columbia University Press, 2004, p. 258

${ }^{25}$ Holmes, Oliver Wendell, «Path of the Law», Harvard Law Review, vol. 10, n. ${ }^{\circ}$ 8, 1896, p. 469

26 Miller, Arthur S., «Reason of State and the Emergent Constitution of Control», Minnesota Law Review, vol. 64, n. ${ }^{\circ}$ 3, 1989, p. 588.

27 Miller, «Notes on the Concept of the Living Constitution», p. 891.

28 Ibid., pp. 891-892.

29 Ibid., p. 892. Miller, «Reason of State and the Emergent Constitution of Control», p. 592.

30 Miller, Arthur S., «Toward a Definition of “The” Constitution», University of Dayton Law Review, vol. 8, n. ${ }^{\circ}$ 3, 1982, p. 660. Miller, Arthur S., «Legal Foundations of the Corporate State», Journal of Economic Issues, vol. 6, n. ${ }^{\circ} 1,1972$, p. 77. 
Un punto sobre el que cabe hacer una consideración es que los jueces deben limitarse a lo que la comunidad habrá de aceptar ${ }^{31}$. Según Miller, los jueces generalmente identifican esos tópicos y actúan en consecuencia ${ }^{32}$. Aún más, el citado autor sugiere que los jueces orientan los resultados de su decisión practicando una fina sensibilidad sobre lo que interesa a la opinión pública, prescindiendo de brindar razones que justifican rigurosamente sus decisiones ${ }^{33}$. Es por ello que, de acuerdo con Miller, solo los profesores, algunos de sus estudiantes y unos pocos abogados especializados se interesan por analizar detenidamente los argumentos utilizados por los jueces que practican el control de constitucionalidad ${ }^{34}$.

Resumiendo, la primera directiva metodológica de una interpretación evolutiva prescribe el deber de examinar las tendencias jurisprudenciales y doctrinarias. Estas se revelan a través de las decisiones que toman los jueces que practican el control de constitucionalidad. Siendo más específicos, un análisis de tendencias conlleva un examen de las decisiones pasadas para con ello inferir la dirección que habrán de tomar las futuras. Se trata, pues, de tomar decisiones judiciales en el presente, sobre la base de experiencias pasadas, para así construir un futuro que colme las expectativas depositadas en él.

\section{SEGUNDA DIRECTIVA INTERPRETATIVA: RECONOCER A LOS ACTORES CONSTITUCIONALES ENCARGADOS DE MATERIALIZAR LAS ASPIRACIONES INCORPORADAS EN LA CONSTITUCIÓN}

Una vez establecido cómo se detectan las tendencias jurisprudenciales y doctrinarias resulta interesante señalar que, de acuerdo con los defensores de la interpretación evolutiva de los derechos, los operadores jurídicos —especialmente los jueces que practican el control de constitucionalidad — no se limitan a seguir el cauce de la historia. Por el contrario, la práctica interpretativa ha de consagrar estándares o principios que las personas sujetas a esa Constitución han de aspirar ${ }^{35}$. En otras palabras, la práctica interpretativa no sigue el curso de la historia, sino que ésta es modelada por la interpretación de los derechos.

${ }^{31}$ Miller, Arthur S., «The Elusive Search for Values in Constitutional Interpretation», Hastings Constitutional Law Quarterly, vol. 6, n. ${ }^{\circ} 2,1979$, p. 501.

32 Ibid., p. 501. Miller, Arthur S., «On the Need for Impact Analysis of Supreme Court Decisions», Georgetown Law Journal, vol. 53, n. ${ }^{\circ}$ 2, 1965, p. 380.

33 Miller, «The Elusive Search for Values in Constitutional Interpretation», p. 502. Miller, Arthur S., «Social Justice and the Warren Court: A Preliminary Examination», Pepperdine Law Review, vol. 11, n..$^{\circ}$ 3, 1983, p. 484. Para un comentario que defiende una interpretación evolutiva de los derechos y mantiene que los jueces deben comprometerse a tomar decisiones orientadas a alcanzar ciertos resultados, Grey, «The Uses of an Unwritten Constitution», p. 236.

${ }_{34}$ Miller, «The Elusive Search for Values in Constitutional Interpretation», p. 502.

35 Miller, «Change and the Constitution», p. 240. 
Lo último plantea un interrogante de suma relevancia, ¿quién es el actor constitucional encargado de consagrar tales aspiraciones? Los intérpretes judiciales. Esta es la respuesta que plantean los defensores de una interpretación viviente de los derechos fundamentales ${ }^{36}$. Sin embargo, es preciso aclarar que los jueces constitucionales amplifican la voz de la comunidad, no la de ellos mismos, como bien ha remarcado Brennan $^{37}$. Los jueces practican la interpretación para buscar concretar el significado de las disposiciones constitucionales al que aspira toda la comunidad. Así, Miller advierte que la constante actualización del texto constitucional que ha realizado Corte Suprema de Justicia de los Estados Unidos de América ha funcionado como una suerte de asamblea constituyente permanente ${ }^{38}$. De esta manera, cada generación ha logrado plasmar su propia Constitución a través de las decisiones judiciales tomadas por los órganos que hacen las veces de intérprete final de las disposiciones constitucionales $^{39}$.

Siguiendo este orden de ideas, Miller advierte que la razón por la que la Constitución de los Estados Unidos de América ha durado tanto radica en la adaptación del tal documento normativo a los problemas de cada época que ha realizado la Corte Suprema ${ }^{40}$. Aún más, la causa que explica la perdurabilidad del texto constitucional estadounidense consiste, pues, en que la Corte Suprema de Justicia opera de una manera cuasi religiosa. La metáfora que propone Miller pretende significar que la Constitución es un objeto de deferencia y adoración para el pueblo ${ }^{41} \mathrm{y}$, en la misma medida, los jueces que practican el control de constitucionalidad hacen las veces de predicadores de ese mensaje cuasi-divino expresado por el texto constitucional ${ }^{42}$.

En efecto, si se asume que la Constitución hace las veces del texto sagrado de la religión civil de los Estados Unidos de América ${ }^{43}$, pues entonces — prosigue Levinson con una buena dosis de ironía - cabe trazar una analogía entre el orden constitucional y la tradición religiosa cristiana: la Constitución le habría confiado la llave del «reino de los cielos» a la Corte Suprema de Justicia ${ }^{44}$. En consecuencia, lo que ate la Corte Suprema a la Constitución resultará parte de ella y lo que desate pues no lo será.

36 Ibid., p. 240.

37 Brennan, «The Constitution of the United States: Contemporary Ratification», p. 434.

38 Miller, «Notes on the Concept of the Living Constitution», p. 885. Miller, «The Elusive Search for Values in Constitutional Interpretation», p. 491.

39 Miller, Arthur S., «Taking Needs Seriously: Observations on the Necessity for Constitutional Change», Washington and Lee Law Review, vol. 41, n. ${ }^{\circ}$ 4, 1984, p. 1251.

${ }_{40}$ Miller, "Change and the Constitution», p. 243. Miller, Arthur S., "The Changing Role of the United States Supreme Court», The Modern Law Review, vol. 25, n. ${ }^{\circ}$ 6, 1962, pp. 641-642.

41 Miller, «On the Need for Impact Analysis of Supreme Court Decisions», p. 391.

42 Miller, «Change and the Constitution», p. 236. Ibid., p. 124.

43 Grey, «The Uses of an Unwritten Constitution», p. 223.

44 Levinson, «"The Constitution” in American Civil Religion», p. 124. La metáfora, además, como Perry lo ha señalado, pretende remarcar que la interpretación constitucional se asemeja más a la interpretación de los textos sagrados que a la interpretación literaria. Perry, Michael J., «The Authority of Text, Tradition, and Reason: A Theory of Constitutional Interpretation», Southern California Law Review, vol. 58, n. ${ }^{\circ} 1 \& 2,1985$, p. 562. Para una detallada explicación de las diferencias entre la interpretación 
Recapitulando, la metáfora religiosa que propone Miller no tiene ninguna clase de pretensión espiritual. Antes bien, el citado autor se ocupa de remarcar la importancia del rol institucional de la Corte Suprema de Justicia como indicador de las líneas jurisprudenciales y doctrinarias que reflejan la evolución de los derechos. Aún más, ese tribunal ejerce el rol de guardián de la conciencia moral de la comunidad; esto es, la Corte sería capaz de articular el sentido último que anima a las disposiciones que integran la Constitución y, por ende, incluso tiene el deber de trascender los consensos actualmente presentes en la sociedad ${ }^{45}$.

\section{TERCERA DIRECTIVA INTERPRETATIVA: RECONOCER LA ESTRUCTURA Y FUNCIONAMIENTO MATERIAL DEL GOBIERNO DEL ESTADO}

\subsection{Primer elemento de la estructura material del gobierno del estado: la práctica de la justicia constitucional como facilitador de la política arquitectónica}

Miller señala que el gobierno del Estado sobrevive no en virtud de los jueces constitucionales, sino a pesar de ellos ${ }^{46}$. Con esto quiere significar que la constitución de un Estado organiza el poder estatal pero, sobre todo, introduce una serie de limitaciones a ese poder ${ }^{47}$. Una vez instituido el gobierno de una comunidad política, en efecto, la función principal que cumple un tribunal constitucional es la de determinar la validez de los cambios constitucionales ${ }^{48}$. En tal sentido, y esto es lo más relevante que pretende señalar Miller, una corte que practica el control de constitucionalidad se ocupa de juzgar la validez de modificaciones a la constitución, ya sea que esto se realice por medio de los canales formales de enmienda o reforma, o bien por medio de la legislación ordinaria, decisiones de la administración pública o de los particulares ${ }^{49}$.

Así, uno de los puntos más relevantes a considerar para la práctica de una interpretación evolutiva pasa por conocer la matriz de organización material de un Estado. Lo último se explica debido a que las metodologías evolutivas suponen una Constitución que se va acomodando a las necesidades y problemáticas actuales que atraviesa el gobierno que la ley fundamental estructura y efectivamente pone en funcionamiento. Es por medio de cambios disruptivos o graduales en la jurisprudencia

de un texto sagrado y la de un texto constitucional, Grey, Thomas C., «The Constitution as Scripture», Stanford Law Review, vol. 37, n. ${ }^{\circ} 1,1984$, pp. 15-17.

${ }^{45}$ Miller, «Change and the Constitution», p. 236.

${ }^{46}$ Ibid., p. 240.

47 Ibid., p. 240.

${ }^{48}$ Bustos Zamora, Geraldine \& Pulido Ortiz, Fabio Enrique, «Momentos constitucionales y legitimidad de los procedimientos de reforma», en Procesos legislativos y ordenamiento constitucional: abordajes teóricos, Gregorio Eljach Pacheco \& et alii (compiladores), Bogotá, CAEL, 2016, pp. 92-105.

${ }^{49}$ Ibid., p. 240. 
constitucional, no a través de procesos de reforma que siguen los cauces formales, como se canalizan los cambios fundamentales que se manifiestan a través de la interpretación evolutiva de la constitución ${ }^{50}$. Dicho de otra manera, si el texto constitucional es preciso interpretarlo a la luz de lo que conlleva implementarlo en la actualidad, pues entonces no hay que atender a la organización formal del gobierno sino a su funcionamiento material.

En tal orden de ideas se puede advertir, en mayor o menor medida, que los gobiernos de las democracias occidentales por lo general asumen un rol activo en la regulación de la economía y la sociedad ${ }^{51}$. Las agencias del Estado ya no se encargan solamente de aquellas funciones que no pueden ser realizadas por la sociedad civil o provistas por el mercado tales como la defensa exterior, seguridad interior, administración de justicia, salud y educación básica ${ }^{52}$. El gobierno, principalmente a través de la dirección del Poder Ejecutivo, es el que marca el tono, el ritmo y la dirección de la economía y, más ampliamente, de la sociedad ${ }^{53}$.

De acuerdo con Miller, en rigor, la historia constitucional estadounidense desde un comienzo adoptó la separación del poder ejecutivo de los otros poderes del gobierno como un medio para asegurar la eficiencia de las decisiones y acciones gubernamentales $^{54}$. De hecho, como bien ha señalado Waluchow, no hay una necesidad conceptual que justifique la separación de poderes, sino que ésta se fundamenta en su conveniencia práctica ${ }^{55}$. En un orden similar de ideas, Miller sugiere que la perdurabilidad de la doctrina de la separación de poderes se debe a que esta nunca ha sido un obstáculo para la cooperación entre ramas del gobierno ${ }^{56}$. Con otras palabras, la funcionalidad práctica de la división de poderes consiste en que haga las veces de facilitador o acelerador de la interacción entre las ramas del gobierno.

La evidencia con que Miller sustenta esta cooperación entre poderes es que —antes y después del «New Deal»- la Corte Suprema solo en dos oportunidades declaró inconstitucional a una delegación de competencias del Poder Legislativo a agencias federales $^{57}$. Ackerman incluso plantea que el estado de cosas no mejoró sensiblemente

50 Ackerman, Bruce, «The Living Constitution», Harvard Law Review, vol. 120, n. ${ }^{\circ}$ 7, 2006, p. 1742. Brennan, Timothy, «Thomas Jefferson and the Living Constitution», The Journal of Politics, vol. 79 , n. ${ }^{\circ}$ 3, 2017, p. 936.

51 Miller, «Legal Foundations of the Corporate State», p. 68. Miller, «The Changing Role of the United States Supreme Court», p. 642.

52 Ugalde, Francisco Valdés, «Concepto y estrategia de la "reforma del Estado” ", Revista Mexicana de Sociología, vol. 55, n. ${ }^{\circ}$ 2, 1993 , p. 317.

53 Miller, «Legal Foundations of the Corporate State», p. 68.

54 Miller, Arthur S., «Separation of Powers: An Ancient Doctrine under Modern Challenge», Administrative Law Review, vol. 28, n. ${ }^{\circ}$ 3, 1976, p. 302.

55 Waluchow, A Common Law Theory of Judicial Review: The Living Tree, p. 40.

56 Miller, «Separation of Powers: An Ancient Doctrine under Modern Challenge», p. 302. Santiago, Alfonso, La Corte Suprema y el control político: función politica y posibles modelos institucionales, Buenos Aires, Ábaco, 1999, pp. 83-84.

57 Miller, «Separation of Powers: An Ancient Doctrine under Modern Challenge», p. 302. 
en la integración Corte Suprema de la nueva era republicana que comenzó con Ronald Reagan. Más aún, este tribunal se habría concentrado más en ratificar las atribuciones del gobierno que en proteger los derechos individuales frente a la acción estatal ${ }^{58}$.

Con todo, Miller remarca que la Corte Suprema desde los años cuarenta ha sido más cooperativa en sus decisiones con relación a la acción de las otras ramas del gobierno que su predecesora ${ }^{59}$. De esta manera, la cooperación entre poderes sería la regla y las tensiones o conflictos no más que excepciones aisladas ${ }^{60}$. En efecto, tal como sugiere Balkin, la Corte Suprema —o, dicho más ampliamente en el contexto estadounidense, el Poder Judicial federal — ha prestado cooperación al gobierno del Estado no tanto mediante sentencias que han apoyado a las decisiones de los otros poderes sino, sobre todo, a través de la inacción de sus jueces en lo que hace a las decisiones que han adoptado los otros poderes del Estado ${ }^{61}$.

De hecho, incluso la conformación de la Corte de Warren que dio lugar a una «revolución de derechos» mediante célebres leading cases tales «Brown v. Board Education of Topeka» ${ }^{62}$ (1954) —en donde se declara que toda segregación racial en establecimientos educativos resulta inconstitucional - y «Katzenbach v. McClung» ${ }^{63}$ (1964) - oportunidad en la que se estableció que la cláusula de comercio interestatal proscribe toda segregación racial en restaurantes_- en paralelo ha guardado un marcado silencio frente a cuestiones que integran la llamada «política de seguridad nacional» ${ }^{64}$.

Aún más, cuando la propia Corte de Warren ha salido de ese silencio fue para convalidar la prevalencia de la seguridad nacional por sobre las libertades civiles como puede advertirse paradigmáticamente en «Barenblatt v. United States of America» ${ }^{65}$ (1959), en donde se estableció que resultaba constitucional una investigación del Congreso de la Nación para indagar si el Prof. Barenblatt estaba afiliado al Partido Comunista. Así, Miller ha planteado que el reconocimiento de derechos civiles de la

${ }^{58}$ Ackerman, Bruce, «Levels of Generality in Constitutional Interpretation--Liberating Abstraction», University of Chicago Law Review, vol. 59, n. ${ }^{\circ}$ 1, 1992, pp. 317-318.

59 Miller, «The Changing Role of the United States Supreme Court», p. 642.

${ }^{60}$ Miller, «Separation of Powers: An Ancient Doctrine under Modern Challenge», p. 303. Balkin, Jack M., «The Roots of the Living Constitution», Boston University Law Review, vol. 92, n. ${ }^{\circ}$ 4, 2012, p. 1137.

${ }^{61} \mathrm{Ibid}$., p. 1138. El caso argentino parece apoyar la tesis de Balkin. En efecto, a partir de un estudio empírico de la Corte Suprema de Justicia de la Nación Argentina basado en una amplia muestra de sentencias 20.000 fallos, de los años 1935-1998 se puede advertir una mayor cantidad de casos que trataron sobre normas de carácter nacional o federal 1149 que provinciales 577 de un total de 1822. Sin embargo, la proporción de normas provinciales declaradas constitucionales fue sensiblemente inferior a nivel provincial 35,4\% mientras que a nivel nacional esa cifra fue bastante superior 54,4\%. Bercholc, Jorge, «Aportes para una selección coherente y congruente de los jueces de un tribunal constitucional. el caso de la corte suprema en la Argentina y sus recientes modificaciones», Revista de derecho público, vol. 1, 2005, pp. 42-43.

62 «Brown v. Board Education of Topeka», 347 U.S. 483 (1954).

63 «Katzenbach v. McClung», 79 U.S. 294 (1964).

${ }^{64}$ Miller, Arthur S., «Pretense and Our Two Constitutions», George Washington Law Review, vol. 54, n. ${ }^{\circ}$ 2, \& 3, 1985, pp. 398-399.

65 «Barenblatt v. United States of America», 360 US 109 (1959). 
Corte de Warren tuvo un objetivo principalmente dirigido a preservar el modelo de Estado corporativo ${ }^{66}$.

Todo lo anterior no implica que los jueces constitucionales han sido enteramente complacientes a las decisiones gubernamentales de los otros poderes del Estado. No estamos necesariamente frente a lo que en la historia de la Corte Suprema de Justicia de la Nación Argentina se ha dado en llamar durante los años noventa como «corte adicta» ${ }^{67}$. Esta visión no se contrapone con la necesidad de que la justicia constitucional realice ajustes y cuestione la dirección de las decisiones tomadas por los poderes políticos. Sin embargo, la intervención de la justicia constitucional no trazará los lineamientos de la política gubernamental, sino que más bien ejercerá una función de control, como bien insinúa Santiago ${ }^{68}$. Esto quiere decir que los jueces se circunscribirán a determinar que la acción gubernamental se despliegue bajo los límites sustantivos trazados por la constitución; esto es, bajo los principios y valores recogidos por la ley fundamental ${ }^{69}$.

Así, la experiencia histórica muestra que incluso en el caso estadounidense ejemplo paradigmático de independencia judicial — la Corte Suprema mayormente ha ratificado a las prácticas políticas de su sistema ${ }^{70}$. Dicho en pocas palabras, los jueces constitucionales han legitimado las decisiones de los poderes políticos especificando lo que los otros poderes pueden hacer, tanto mediante su silencio como marcando positiva y activamente los límites de la acción gubernamental ${ }^{71}$.

\subsection{Segundo elemento de la estructura material del gobierno del estado: el surgimiento del nuevo orden constitucional basado en un modelo de Estado corporativo}

Situados en este contexto de ideas, es preciso advertir que actualmente ya no estamos frente a un gobierno del Estado que algunos llamarían estado «gendar-

66 Ibid., p. 398.

${ }^{67}$ Esta visión de la Corte Suprema como un órgano meramente ratificador de las decisiones de los poderes políticos en la década de los noventa durante la administración de Carlos Menem fue incluso recogida públicamente por Gustavo Béliz, el Ministro de Justicia del gobierno siguiente. Ruibal, Alba M., «La sociedad civil en el proceso de reformas a la Corte Suprema argentina», Revista Mexicana de Sociología, vol. 70, n. ${ }^{\circ}$, 2008, p. 749. Sin embargo, esta percepción ha sido refutada por los estudios empíricos de Bercholc, quien ha reunido evidencia que permite concluir que esa Corte ha sido la que mayor cantidad de inconstitucionales sobre normas constitucionales dictadas por un gobierno actual. Bercholc, Jorge, «Aportes para una selección coherente...», pp. 42-43.

${ }^{68}$ Santiago, La corte suprema y el control político: función política y posibles modelos institucionales, p. 84

69 Oyhanarte, Julio César, Poder político y cambio estructural en la Argentina: un estudio sobre el estado de desarrollo, Buenos Aires, Paidós, 1969, pp. 58 y ss.

${ }^{70} \mathrm{La}$ tendencia a desplazar el eje de las cuestiones políticas no justiciables a las decisiones judiciales que establecen «remedios estructurales» parece congfirmar un abandono de la doctrina de la separación de poderes del Estado cual si fueran compartientos estancos, Risso Ferrand, Martín José, «Dificultades en la protección judicial de los derechos humanos», Anuario Iberoamericano de Justicia Constitucional, n. ${ }^{\circ}$ 20, 2016, p. 346 . DOI: http://dx.doi.org/10.18042/cepc/aijc.20.12.

71 Balkin, «The Roots of the Living Constitution», p. 1137. 
me» o «liberal», el cual caracterizó al nacimiento del movimiento constitucionalista a finales del siglo XVIII o principios del S. XIX. Tampoco estamos frente a un estado de «bienestar», «activista» o, mejor dicho, ya hemos superado aquel orden constitucional anclado en el New Deal cuyo horizonte de sentido, como afirma Tushnet, ha de buscarse en el igualitarismo ${ }^{72}$. En otras palabras, si el Estado liberal se afanaba por concretar la libertad en sentido negativo, el modelo de Estado de bienestar que le siguió pretendió alcanzar la igualdad, pero este ya se habría dejado atrás.

En efecto, el Estado bienestar se anclaba en una concepción filosófica que se dio en llamar «igualitarismo» y que se ha estructurado, de acuerdo con Rawls, sobre dos pilares fundamentales, (1) cada persona tiene igual derecho a un completo goce de los derechos y libertades fundamentales, lo que resulta compatible con algo semejante para todos los seres humanos. 2) Las desigualdades sociales y económicas se han de remediar mediante: a) un acceso a los puestos laborales que esté abierto a todos bajo una igualdad de oportunidades y, b) beneficiar a los miembros más desaventajados de la sociedad ${ }^{73}$. Naturalmente, tal como Rawls lo advierte y más recientemente Tushnet, hubo desacuerdos profundos sobre cómo alcanzar tales metas ${ }^{74}$. Con todo, este modelo se habría agotado o, mejor dicho, se ha reformulado parcialmente porque hoy estamos frente a un nuevo orden constitucional ${ }^{75}$.

Es justo destacar que esta reformulación es tan solo parcial porque, como bien señala Miller, siempre se ha estado frente a un estado «fuerte» o, mejor dicho, el Estado ha sido permanentemente tan intenso como las circunstancias de cada época lo han requerido ${ }^{76}$. Dicho de otra manera, no es que el gobierno comienza a actuar positiva o activamente a partir del New Deal, sino que siempre lo ha hecho, aunque

72 Tushnet, Mark V., The New Constitutional Order, New Jersey, Princenton University Press, 2003, p. 9. En un sentido similar, se ha sostenido que la adopción de una metodología interpretativa evolutiva o viviente ha implicado profundas consecuencias en el desarrollo de derechos que se desprenden del principio de igualdad. L'Heureux-Dube, Claire, «The Legacy of the Persons Case: Cultivating the Living Tree’s Equality Leaves», Saskatchewan Law Review, vol. 63, n. ${ }^{\circ}$ 2, 2000, p. 396. Una aplicación reciente de la doctrina del igualitarismo como base para remediar la pobreza y alcanzar una igualdad de oportunidades, Ferreyra, Gustavo R., «Gobernar es igualar: isonomía, oportunidades y justicia social en la Argentina», Revista de Derecho Politico, vol. 99, 2017, pp. 435-473.

73 Rawls, John, «Justice as Fairness: Political Not Metaphysical», Philosophy E Public Affairs, vol. 14, n. ${ }^{\circ}$ 3, 1985, p. 227. En un sentido similar, Miller, «Separation of Powers: An Ancient Doctrine under Modern Challenge», p. 310. Fiss, Owen M, «Groups and the Equal Protection Clause», Philosophy \& Public Affairs, vol. 5, n. ${ }^{\circ} 2,1976$, pp. 159-161. Una aplicación de estas ideas orientada a demostrar que las políticas globales de carácter social no apuntan sino a cubrir un mínimo de las necesidades básicas, Páez, Pedro Nel; Jiménez William, Guillermo \& Buitrago, Jenny Danna, «Necesidades humanas y política social», Revista Republicana, vol. 22, 2017, pp. 131-158. DOI: http://dx.doi.org/10.21017/ Rev.Repub.2017.v22.a23

${ }^{74}$ Rawls, «Justice as Fairness: Political Not Metaphysical», p. 227. Tushnet, The New Constitutional Order, p. 9.

75 Ibid., pp. 9-32.

${ }^{76}$ Miller, «Separation of Powers: An Ancient Doctrine under Modern Challenge», p. 303. 
con diversas intensidades ${ }^{77}$. Esto implica que, los llamados «derechos de contenido negativo» conllevan una serie de acciones positivas por parte del gobierno, que de ningún modo cabe apreciar como meramente negativas. Así, por ejemplo, la libertad de contratar requiere de la organización y mantenimiento de tribunales que administran justicia para concretar eficazmente la obligatoriedad de los vínculos contractuales ${ }^{78}$. En tal sentido lo que resulta novedoso de la concepción del Estado a partir del New Deal resulta ser más bien la medida, nivel o intensidad de la acción positiva o intervencionista del gobierno del Estado. En este orden de ideas, la Constitución pasó de funcionar centralmente como una serie de limitaciones a la acción política arquitectónica para terminar siendo un listado expansivo de competencias gubernamentales ${ }^{79}$. Ackerman sugiere que la carta de derechos — Bill of Rights_ está limitada por el entendimiento que el gobierno hace de los principios que subyacen a su «carta de poderes»—Bill of Powers - Dicho de otro modo, parece que la vigencia de los derechos fundamentales depende de la comprensión que el gobierno tiene de sus propias atribuciones.

El modelo de gobierno que le ha seguido al Estado intervencionista o de bienestar es el llamado «nuevo orden constitucional» ${ }^{80}$. Lo específico de este nuevo régimen no pasa tanto porque el gobierno ha dejado de intervenir en la sociedad o en la economía, sino porque su punto de apoyo ha dejado de ser el electorado y, en cambio, ahora se orienta decisivamente a alcanzar la consolidación del gobierno mismo ${ }^{81}$. Se trata, pues, de un gobierno que responde a sus propios intereses. Esto se debe a que estamos frente a lo que Miller hace un tiempo había llamado como «Estado Corporativo»; esto es, un modelo de dirección del Estado en que se entremezclan el gobierno y las corporaciones privadas ${ }^{82}$.

Más en concreto, se habrían fusionado los poderes económicos y el poder político. Esto incluso se ha expresado jurídicamente, por ejemplo, a través de comités o agencias compuestas tanto por integrantes del sector público como también por representantes de las corporaciones del sector privado ${ }^{83}$. Y, sobre todo, lo más específico del Estado corporativo pasa por el hecho de que la unión entre corporaciones y gobierno resulta más significativa que la mera suma de las partes ${ }^{84}$. Esto ha llevado al propio Miller a resaltar que el Estado corporativo ya no se orienta princi-

77 Sunstein, Cass R., «State Action is Always Present», Chicago Journal of International Law, vol. 3, n. ${ }^{\circ}$ 2, 2002, p. 466. Holmes, Stephen \& Cass R Sunstein, El costo de los derechos: por qué la libertad depende de los impuestos, trad. Stella Mastrangelo, Buenos Aires, Siglo XXI, 2011, p. 33.

78 Sunstein, «State Action is Always Present», p. 466.

79 Miller, «Separation of Powers: An Ancient Doctrine under Modern Challenge», p. 310.

80 Tushnet, The New Constitutional Order, capítulo 1.

81 Ibid., p. 12.

82 Miller, «Reason of State and the Emergent Constitution of Control», p. 610. Miller, «Pretense and Our Two Constitutions», p. 393. Miller, Arthur S., «Public Confidence in the Judiciary: Some Notes and Reflections», Law and Contemporary Problems, vol. 35, n. ${ }^{\circ} 1,1970$, p. 71.

83 Miller, «Legal Foundations of the Corporate State», p. 60.

84 Ibid., p. 60. 
palmente a satisfacer a las necesidades de los individuos, sino a fortalecer esta alianza entre corporaciones y gobierno ${ }^{85}$. Dicho de otra manera, se trata de un gobierno que se sirve a sí mismo.

\section{CUARTA DIRECTIVA INTERPRETATIVA: PROYECTAR LAS CONSECUENCIAS (POLÍTICAS) DE LA DECISIÓN JUDICIAL SOBRE LA ESTRUCTURA SOCIAL}

La cuarta directiva de una interpretación evolutiva de los derechos consiste en proyectar las consecuencias de los resultados interpretativos sobre la estructura social ${ }^{86}$. Se trata, como diría Lombardi Vallauri, de una «metodología de resultados», es decir, la selección de la técnica interpretativa evolutiva se funda en la realización de una serie de valores políticos a realizar, una y otra vez, en cada decisión judicial concreta $^{87}$. Por lo tanto, la interpretación no es un tipo de razonamiento estricta o rigurosamente lógico ${ }^{88}$.

Es preciso subrayar que de lo anterior no se sigue un desdén a la contribución de la lógica a la ciencia del Derecho, sino que en una decisión judicial, como bien sintetizó Frankfurter al evaluar el legado intelectual del justice Holmes, el intérprete judicial debe articular una sabiduría sociológica y una lógica consistente al momento de practicar el control de constitucionalidad ${ }^{89}$. En consecuencia, la lógica cumple una función meramente instrumental al desarrollo o evolución del derecho constitucio$\mathrm{nal}^{90}$. Con otras palabras, la lógica sirve como herramienta para tomar decisiones judiciales capaces de resolver coherentemente los desafíos del presente mediante una conexión con las experiencias pasadas.

Así, la orientación política de la práctica interpretativa, como bien advierte Dworkin, es inevitable que remita a una cierta moralidad política ${ }^{91}$. El juez constitucional solo es capaz de resolver los casos difíciles mediante una orientación teleoló-

85 Ibid., p. 61.

${ }^{86}$ Miller, «Legal Foundations of the Corporate State», p. 73. Strauss, The Living Constitution, p. 34.

87 Lombardi Vallauri, Luigi, Corso di Filosofia del Diritto, Padova, CEDAM, 1981, pp. 53-54. En un sentido similar, Ely afirma que la aplicación de toda metodología interpretativa necesariamente está influenciada por elecciones políticas Ely, John Hart, «Constitutional Interpretivism: Its Allure and Impossibility», Indiana Law Journal, vol. 53, n. ${ }^{\circ} 3,1977$, p. 403.

${ }^{88}$ Lombardi Vallauri, Corso di Filosofia del Diritto, pp. 67-68.

${ }^{89}$ Frankfurter, Felix, «Mr. Justice Holmes and the Constitution a Review of His Twenty-Five Years on the Supreme Court», Harvard Law Review, vol. 41, n. ${ }^{\circ}$ 2, 1927, pp. 139-147.

90 Pound, Roscoe, «Natural Natural Law and Positive Natural Law», Natural Law Forum, vol. 5, 1960, p. 75. Del mismo autor, "Canons of Procedural Reform», Annual Report of the American Bar Association, vol. 49, 1926, p. 291.

91 Dworkin, Ronald, «The Arduous Virtue of Fidelity: Originalism, Scalia, Tribe, and Nerve», Fordham Law Review, vol. 65, n. ${ }^{\circ}$ 4, 1996, pp. 1249-1250. En un sentido similar, Serna, Pedro, «Presupuestos programáticos para la elaboración de un concepto hermenéutico-ontológico de Derecho», Revista Portuguesa de Filosofia, vol. 70, n. ${ }^{\circ}$ 2/3, 2014, pp. 223-224. 
gica basada en consideraciones de naturaleza moral. La Constitución, pues, incorpora principios morales abstractos cuyo sentido y aplicación a casos concretos se devela por medio de la interpretación ${ }^{92}$. En efecto, Dworkin afirma que la lectura moral de la Constitución resulta inevitable y no meramente deseable. En otras palabras, ante casos de normas que se expresan mediante un lenguaje vago se incurre necesariamente en una lectura moral de la Constitución.

Así, el intérprete ha de construir una coherente, principialista y persuasiva interpretación de alguna particular disposición constitucional, tomando a la Constitución como un todo, junto con la historia que se desplegó bajo el amparo de tal constitución ${ }^{93}$. Esto posibilita alcanzar lo que Dworkin designa como «integridad constitucional» ${ }^{94}$. Un punto importante por remarcar de la propuesta dworkiniana es que los miembros de la comunidad de intérpretes - $p$. e , jueces, abogados, ciudadanos - deben procurar enlazar sus respuestas con la práctica y tradición del sistema en que están insertados. Aún más, los intérpretes deben tomar conjuntamente el texto, la historia y la práctica constitucional para elaborar respuestas capaces de reflejar los compromisos asumidos por cada nación al darse su ley fundamental ${ }^{95}$.

Ahora bien, ¿cómo explica Dworkin los giros copernicanos en la doctrina judicial de un sistema constitucional? ¿Si los intérpretes constitucionales se encuentran atados por el texto, la historia y la práctica constitucional cómo explicar y justificar precedentes como «Brown v. Board of Education»? La respuesta del profesor de la Universidad de Nueva York es que la práctica constitucional de los Estados Unidos de América venía ignorando estándares generales de igualdad que proscribían toda discriminación que no sirviera directamente a un legítimo interés estatal ${ }^{96}$. Por lo tanto, Brown no fue tanto un giro copernicano en la jurisprudencia de la Corte Suprema, sino una lectura más integral, completa y coherente de la práctica constitucional. Dicho de otro modo, los jueces constitucionales habrían descubierto que la segregación racial era inaceptable mediante la aplicación de una meticulosa «integridad constitucional».

Sin embargo, es preciso subrayar que el propio Dworkin aclara que no toda interpretación constitucional debe aplicar una «lectura moral», sino que esta procede exclusivamente ante disposiciones normativas que remiten a un lenguaje moral de naturaleza abstracta. En efecto, la lectura moral de la constitución no procede frente a disposiciones redactadas mediante formulaciones lingüísticas bien específicas ${ }^{97}$ como, por ejemplo, aquella norma que prescribe el deber de haber cumplido 30 años

92 Fleming, James E., «Living Originalism and Living Constitutionalism as Moral Readings of the American Constitution», Boston University Law Review, vol. 92, n. ${ }^{4}$, 2012, p. 1173.

93 Dworkin, «The Arduous Virtue of Fidelity: Originalism, Scalia, Tribe, and Nerve», p. 1250.

94 Ibid., p. 1250.

95 Ibid., p. 1254.

96 Ibid., p. 1254.

97 Dworkin, Freedom's Law: The Moral Reading of the American Constitution, p. 8. 
para ser senador ${ }^{98}$, o que el mandato del presidente durará cuatro años ${ }^{99}$. Por ende, si la disposición constitucional no es abstracta, pues entonces no existe ningún espacio para una remisión a la moral. Se podría resumir lo dicho a través de la siguiente máxima: «a mayor nivel de abstracción, mayor necesidad de practicar una lectura moral de la constitución».

\section{QUINTA DIRECTIVA INTERPRETATIVA: ASUMIR LA INVENCIBILIDAD DEL TEXTO CONSTITUCIONAL Y LA TRADICIÓN FRENTE A LAS ASPIRACIONES, IDEALES O VALORES ACTUALES}

La quinta directiva interpretativa de una metodología viviente o evolutiva supone una elección frente a la derrota o prevalencia del texto constitucional ante las aspiraciones, ideales o valores contemporáneos. A tal derrota del texto, siguiendo el trabajo de Michael S. Moore, la llamaremos «interpretación evolutiva sustitutiva» y a la negación de tal derrota, en cambio, la designaremos como «interpretación evolutiva complementaria» ${ }^{100}$. Dworkin plantea que la anterior categorización no se corresponde con la realidad porque, de un modo u otro, todos los que interpretan la Constitución afirman que sus prácticas se apoyan en el texto constitucional. Sin embargo, el punto de partida de una interpretación basada en el texto no es la cuestión problemática, sino la posibilidad de concluir que las disposiciones constitucionales son un elemento completamente prescindible en caso de conflictos con los valores, ideales y aspiraciones morales de la hora presente.

La interpretación evolutiva sustitutiva es más infrecuente que la versión complementaria. Esta última, en efecto, consiste en asumir que es deseable complementar el texto, tradición y práctica interpretativa constitucional con las aspiraciones, valores e ideales actuales ${ }^{101}$. Como sugiere Waluchow en su defensa de la interpretación viviente, la supremacía de la Constitución no rechaza, sino que asume como conveniente que la interpretación de las disposiciones iusfundamentales remita a las consideraciones sustantivas actuales sobre justicia y moralidad política actual ${ }^{102}$. Dicho de otra manera, es posible enlazar un respeto a la supremacía de la ley fundamental con las aspiraciones morales que nutren a la práctica interpretativa constitucional.

Así, la interpretación evolutiva de tipo complementaria supone una concepción robusta de la función del control de constitucionalidad que de ningún modo pretende negar la relevancia de texto constitucional. El punto que caracteriza a esta versión de la interpretación evolutiva consiste en la remisión a elementos extra normativos

\footnotetext{
98 Constitución de los Estados Unidos de América, Artículo Uno, Tercera Sección, inciso 3.

99 Constitución de los Estados Unidos de América, Artículo Dos, Primera Sección, inciso 1.

100 Moore, «Do We Have an Unwritten Constitution?», p. 115.

101 Ibid., p. 115.

102 Waluchow, «Constitutions as Living Trees: An Idiot Defends», pp. 212-213.
} 
— valores y aspiraciones no contenidas en el texto constitucional—, que han de ser tenidos en cuenta como un elemento auxiliar al momento de interpretar la Constitución ${ }^{103}$. No obstante, esto solo procede cuando el texto constitucional se caracteriza por su vaguedad. En consecuencia, la apertura a complementar el texto constitucional con ideales y valores actuales depende del grado de vaguedad de las disposiciones normativas a interpretar.

De hecho, en los casos más relevantes del derecho constitucional, según plantea Grey, el análisis del texto constitucional desempeña un papel marginal. Lo que se discute sustancialmente en los grandes casos no descansa tanto en el sentido de las disposiciones normativas como en los valores e ideales que se atribuyen al texto que integra la Constitución ${ }^{104}$. De esta manera, las disposiciones constitucionales vagas son vistas como la fuente de legitimidad para que los intérpretes judiciales elaboren, desarrollen y expliquen el contenido de valores compartidos en un particular sistema constitucional $^{105}$. Más aún, los redactores de una Constitución suelen tener la sabiduría y prudencia de dejar en manos de los jueces una considerable cuota de poder para determinar el contenido de los derechos fundamentales sin una restricción significativa del texto y la historia ${ }^{106}$.

Situados en este orden de ideas es posible apreciar que lo propio de esta metodología evolutiva complementaria es que la caracterización de tales valores e ideales hace referencia a consideraciones morales sobre lo que actualmente se entiende como justo ${ }^{107}$. Se trata, pues, de una concepción de la constitución que reconoce derechos fundamentales, pero no mediante el establecimiento de puntos fijos de acuerdo moral al momento de su adopción. Antes bien, las cartas de derechos serían comparables a un «árbol viviente que crece y se adapta de acuerdo con sus límites naturales» ${ }^{108}$. Con otras palabras, las disposiciones constitucionales solo hacen las veces de la semilla a partir del cual se desarrolla el árbol que es la Constitución materialmente vigente en el Estado.

Ahora bien, ¿cómo se hace para reconocer aquellos valores, aspiraciones e ideales morales que complementan al texto constitucional? Gargarella advierte que la respuesta que suele darse al anterior interrogante consiste en mirar alrededor y replicar lo que otros intérpretes de la Constitución afirman sobre una determinada cuestión ${ }^{109}$. Con todo, y más allá de lo que efectivamente realizan muchos jueces, la interpretación evolutiva de los derechos puede descansar en una metodología interpretativa basada en lo que Dworkin ha entendido por «derecho como integridad». De hecho, no hace faltar

${ }^{103}$ Grey, «The Uses of an Unwritten Constitution», p. 211. Grey, «Do We Have an Unwritten Constitution?», p. 706.

104 Ibid., p. 706.

105 Ibid., p. 709.

106 Ibid., p. 714.

107 Waluchow, «Constitutions as Living Trees: An Idiot Defends», p. 222.

108 «Edwards», p. 136.

109 Gargarella, Roberto, «Interpretation and Democratic Dialogue», Revista da Faculdade de Direito UFPR, vol. 60, n. ${ }^{\circ}$ 2, 2015, p. 52. DOI: http://dx.doi.org/10.5380/rfdufpr.v60i2.42197 
asumir aquella discutible tesis dworkiniana de que todas las interpretaciones del Derecho, frente a los casos difíciles, suponen una lectura moral que necesariamente integra el texto, con la tradición y la práctica. Dejando de lado la inevitabilidad de una lectura integral del Derecho tal como la entiende Dworkin, lo cierto es que tal metodología interpretativa permite articular un respeto por el texto, las aspiraciones morales actuales y la historia que atraviesa a la práctica interpretativa del sistema constitucional.

\section{LOS PRESUPUESTOS SEMÁNTICOS DE UNA INTERPRETACIÓN EVOLUTIVA DE LOS DERECHOS: UNA TEORÍA DEL SIGNIFICADO DE NATURALEZA CONVENCIONALISTA}

\subsection{El planteamiento del problema: una teoría semántica de naturaleza convencionalista}

A grandes rasgos, existen dos posibilidades o tipologías de presupuestos semánticos en los que descansa la interpretación jurídica —en general—: el convencionalismo o realismo semántico. Este último se apoya en la prioridad de la referencia por sobre el aspecto social o convencionalmente construido ${ }^{110}$. El convencionalismo semántico, en cambio, es la teoría dominante en lo que hace a la naturaleza y alcance de los conceptos a partir de los cuales se desarrolla la interpretación jurídica. Esta teoría semántica afirma una inversión de prioridades respecto del realismo semántico. Así, siguiendo la senda de Austin, el convencionalismo sostiene una teoría del significado por la cual se afirma que la extensión —o referencia — de los conceptos depende principalmente de construcciones sociales de significado o, dicho en términos más técnicos, «convenciones lingüísticas» ${ }^{111}$. Sin embargo, es preciso aclarar que no se trata de que los conceptos son construidos entera o completamente por consensos o convenciones semánticas, sino que tales convenciones tienen prioridad sobre la referencia de los conceptos ${ }^{112}$.

110 Moore, «Justifying the Natural Law Theory of Constitutional Interpretation», p. 2091.

111 Austin, «How to Talk. Some Simple Ways», p. 229. Para un trabajo que defiende la idea de que la regla de reconocimiento del Derecho depende de una convención social, Ortega García, «The Conventionality of Law», p. 246.

112 Laise, Luciano D., El poder de los conceptos: convenciones semánticas y objetividad referencial en la interpretación constitucional originalista, Méjico D. F., Porrúa, 2017, p. 13. En un sentido similar, Cianciardo, sobre la base de la obra de Javier Hervada, afirma que los derechos humanos tienen una dimensión cultural pero su juridicidad no depende enteramente de su reconocimiento social. Tales derechos, pues, son anteriores a su formalización mediante tratados internacionales de derechos humanos o carta de derechos incorporadas en textos constitucionales. Cianciardo, Juan, «Problemas que una nota esencial de los derechos humanos continúa planteando a la filosofía del Derecho», Persona y Derecho, vol. 76, n. ${ }^{\circ} 2$, 2017, p. 87 y ss. DOI: https://doi.org/10.15581/011.76.83-91. Para una aproximación monográfica a los presupuestos epistemológicos del citado Javier Hervada, Herrera Pardo, Camila, Aproximación a los fundamentos científicos y filosóficos del iusnaturalismo realista de Javier Hervada, Pamplona, EUNSA, 2016, passim. 
No obstante, el convencionalismo semántico presenta dos dificultades de las que no puede escapar: i) el regreso al infinito de interpretaciones; ii) la confusión entre discrecionalidad y arbitrariedad judicial. Los próximos dos subepígrafes se abocarán a tales cuestiones.

\subsection{El regreso al infinito de interpretaciones}

El convencionalismo semántico no es capaz de evitar un regreso al infinito de interpretaciones. Porque la reducción del significado de los conceptos al uso lingüístico compartido incurre necesariamente en una cadena infinita de usos lingüísticos. Esto podría ser ejemplificado del siguiente modo: el significado del «esclavitud» se ha de comprender evolutivamente y no equivale a «la existencia de un título de propiedad sobre el esclavo, sino el ejercicio de poderes vinculados con la propiedad que se traducen en la destrucción o anulación de la personalidad jurídica del ser humano» ${ }^{113}$.

Entonces, alguien podría preguntarse, ¿qué significa «poderes vinculados con la propiedad»; «destrucción o anulación de la personalidad jurídica del ser humano» y, finalmente, ¿qué quiere decir «destrucción o anulación de la personalidad jurídica del ser humano»? A estos interrogantes, desde una semántica convencionalista, solo se podría responder que cada una de tales cuestiones se explica por medio de una referencia a la convención semántica o uso lingüístico compartido «b2». Luego, una operación similar ocurriría con el significado de «b2», ya que este designaría al uso generalizado «b3». De manera que la pregunta por el significado de una expresión jurídica haría referencia a otros conceptos que, a su vez, remitirían a otros nuevos conceptos, y así hasta el infinito ${ }^{114}$.

Este encadenamiento infinito de convenciones semánticas supone una concepción del lenguaje que bloquearía todo acceso a una realidad que no dependa de convenciones lingüísticas. Se trataría de una concepción del lenguaje puramente autorreferencial; esto es, un lenguaje que no hace más que discurrir sobre sí mis$\mathrm{mo}^{115}$. Además, tal carácter autorreferencial del lenguaje entrañaría la imposibilidad de que la determinación del significado de un enunciado jurídico resulte capaz de detenerse en una formulación específica y definitiva. En efecto, tal determinación del significado, desde la perspectiva convencionalista, constituiría un pensamiento iterativo; esto es, un pensamiento incapaz de alcanzar alguna dimensión aprehensible de la realidad. Por lo tanto, se trataría de un pensamiento que se circunscribe exclusivamente a reflexionar cómo se piensa lo que se piensa ${ }^{116}$. Recapitulando, el

113 Corte I.D.H., Sentencia «Trabajadores de la Hacienda Brasil Verde vs. Brasil», del 20 octubre de 2016, Serie C, N. $318 . \$ 259$.

114 Zambrano, Pilar, «Fundamental Principles, Realist Semantics, and Human Action», Rechtstheorie, vol. 46, 2015, pp. 332-333. DOI: https://doi.org/10.3790/rth.46.3.323.

115 Laise, El poder de los conceptos: convenciones semánticas y objetividad referencial en la interpretación constitucional originalista, p. 73.

116 Inciarte, Fernando, El reto del positivismo lógico, Madrid, RIALP, 1974, pp. 56-57. 
pensamiento iterativo resulta ser una operación intelectual pura y exclusivamente remitente que no puede ir más allá de la descripción de una cadena infinita de usos lingüísticos.

\subsection{El ocaso de la distinción entre discrecionalidad y arbitrariedad judicial}

En segundo término, el convencionalismo semántico no es capaz de evitar una confusión entre discrecionalidad y arbitrariedad interpretativa. En hilo con la anterior dificultad, una semántica convencionalista implica que la dilucidación del significado de los enunciados jurídicos remite a convenciones lingüísticas, estas a su vez a otras convenciones, y así hasta el infinito, como se ha explicado anteriormente. Frente a tal dificultad, se podría apelar a un acto de decisión del juez que interrumpiría tal cadena de remisiones en algún eslabón determinado. No obstante, desde el convencionalismo semántico no podrían darse razones para justificar tal corte o interrupción en la cadena de remisiones ${ }^{117}$. Se trataría, a lo sumo, de un cierre arbitrario a este infinito encadenamiento de interpretaciones.

En efecto, desde las coordenadas en las que se ubica el convencionalismo semántico, los criterios mínimos para reconocer un uso correcto de los conceptos no pueden ser delimitados sino en virtud de una decisión arbitraria; esto es, una decisión que no se justifica más que en la remisión a una serie de prácticas compartidas y no en virtud de su intrínseca racionalidad para concretar alguna dimensión básica o fundamental del desarrollo de la persona humana y su dignidad.

Así, no sería posible distinguir una decisión judicial arbitraria de una discrecional; es decir, de una elección racional entre diversas alternativas «abiertas» ${ }^{118}$. Dicho en otras palabras, si el significado de las disposiciones jurídicas —en general - y de las de tipo constitucional —en particular - depende prioritariamente de convenciones lingüísticas, el único modo posible de escapar de un regreso al infinito es apelando a una decisión judicial voluntarista exenta de todo control racional que atribuye un determinado significado al enunciado normativo que se interpreta $^{119}$.

117 Laise, El poder de los conceptos: convenciones semánticas y objetividad referencial en la interpretación constitucional originalista, pp. 50-51.

118 Etcheverry, Juan B., «Discrecionalidad Judicial. Causas, Naturaleza y Límites», Teoría y Derecho: revista de pensamiento jurídico, vol. 15, 2014, p. 154. Etcheverry, Juan B., «Rule of Law y discrecionalidad judicial: compatibilidad y recíproca limitación», Revista Derecho del Estado, n. ${ }^{\circ}$ 38, 2017, p. 6. DOI: https://doi.org/10.18601/01229893.n38.01. Para un tratamiento de la discrecionalidad en el contexto de la doctrina del precedente judicial, Pulido Ortiz, Fabio Enrique, Jueces y reglas: la autoridad del precedente judicial, Bogotá, Editorial Universidad de La Sabana, 2018, p. 200 y ss.

119 Laise, El poder de los conceptos: convenciones semánticas y objetividad referencial en la interpretación constitucional originalista, p. 51. 


\section{A MODO DE CONCLUSIÓN}

La interpretación evolutiva o viviente de los derechos se puede desglosar en dos clases de metodologías interpretativas. (i) Una versión que podríamos llamar «sustitutiva», en tanto suplanta el texto y la tradición interpretativa por una remisión a valores, aspiraciones e ideales morales contemporáneos. (ii) Una versión que podría designarse como «complementaria» en tanto suplementa, pero no reemplaza, el texto y la tradición mediante una remisión a valores, aspiraciones e ideales morales contemporáneos.

Una de las principales contribuciones de este trabajo consistió en la sistematización de las directivas interpretativas de las metodologías evolutivas. En tal sentido, se planteó una reconstrucción de la interpretación viviente o evolutiva centrada en cinco directivas: 1) detectar a las tendencias jurisprudenciales y doctrinarias; 2) reconocer el rol institucional de los actores encargados de interpretar los derechos; 3) identificar la estructura formal y el funcionamiento material del gobierno del estado; 4) proyectar las consecuencias del resultado interpretativo proyectado sobre el interés general de la comunidad política; 5) asumir la invencibilidad del texto y la tradición interpretativa frente a las aspiraciones, valores e ideales morales actuales. Esta última directiva resulta de especial trascendencia porque en la medida en que se asumiese la posibilidad de derrota del texto constitucional, y la tradición interpretativa que se sigue de él, la ley suprema ya no sería la ley suprema del país, sino las aspiraciones e ideales contemporáneos que se atribuyen a ella.

Sin embargo, las distintas versiones de la interpretación evolutiva de los derechos no solo se enfrentan con el reto de mantener la supremacía de la Constitución. En efecto, tanto la versión complementaria como la sustitutiva presuponen una semántica convencionalista. Esta teoría supone que el significado de los derechos depende de conceptos que refieren prioritariamente a una construcción social del significado. Una fundamentación semántica de la interpretación viviente que descanse en presupuestos que asignen una prevalencia al aspecto socialmente construido por sobre la referencia los conceptos terminaría incurriendo en un regreso al infinito de interpretaciones; es decir, el significado de un concepto constitucional se dilucidaría a la luz de una convención semántica, pero el significado de esta, a su vez, remitiría a otra convención y así hasta el infinito.

Esta remisión de una convención semántica a otra podría solucionarse mediante una decisión del intérprete constitucional, pero en la medida que se asume una semántica convencionalista no se podrían brindar razones que justifiquen la práctica interpretativa. Estaríamos frente a un acto de decisión necesariamente voluntarista. Siguiendo este camino resulta inevitable la confusión entre arbitrariedad y discrecionalidad judicial.

En efecto, resulta imprescindible que toda interpretación evolutiva o viviente de los derechos fundamentales no descanse exclusivamente en consensos o convenciones semánticas. Una decisión judicial capaz de proporcionar un resultado interpretativo 
justificado requiere necesariamente de una concepción semántica del lenguaje constitucional que subordine el significado de las normas y disposiciones constitucionales a bienes o dimensiones básicas del desarrollo de la personalidad humana. Solo de esta manera resulta posible articular el respeto al texto, la inserción en la tradición constitucional y, a la vez, una adaptación a los valores y aspiraciones morales actuales. El desarrollo de esta semántica constitucional anclada en una prioridad de la referencia por sobre las convenciones semánticas es una cuestión que merece un tratamiento detenido en futuros trabajos. Esto último es, al modo de ver de este autor, uno de los desafíos más relevantes de la teoría de la interpretación constitucional en la hora presente.

Title:

The Living Constitutionalism. Interpretive Method, Semantic Presuppositions and Difficulties

\section{Summary:}

Setting the stage: the challenge of systematizing the nature and semantic presuppositions of evolving interpretive methodologies. I. First interpretive instruction: to identify the jurisprudential and doctrinal trends. I. Second interpretive instruction: to recognize the constitutional agents who construct aspirations incorporated in the Constitution. III. Third interpretive instruction: to recognize the formal structure and actual function of government of the state. IV. Fourth interpretive instruction: to cast (political) consequences of judicial decision on the social structure. V. Fifth interpretive instruction: to assume the indefeasibility of constitutional text and tradition before current aspirations, ideals and values. VI. Semantic presuppositions of evolving interpretation of rights: a conventionalist semantic theory. VII. Outline for overcoming the difficulties of conventional semantics applied to evolving interpretation of rights. Conclusions.

\section{Resumen:}

Las metodologías evolutivas o «vivientes» se utilizan con frecuencia en las prácticas interpretativas de los tribunales constitucionales e internacionales. Sin embargo, tanto las defensas como las críticas de tales metodologías rara vez caracterizan o sistematizan sus directivas interpretativas. El objetivo de este trabajo apunta, en primer lugar, a revertir tales insuficiencias mediante una reconstrucción de los pasos metodológicos que plantean los defensores del constitucionalismo viviente. Luego, se procederá a 
identificar los presupuestos semánticos de las metodologías evolutivas. Los resultados de esta investigación pretenden argumentar que tales presupuestos se caracterizan por una teoría del significado radical o eminentemente convencional; esto es, la extensión de los derechos fundamentales se apoyaría más en consensos sociales que en los bienes constitucionales que tales derechos protegen. Esta dificultad presenta especial relevancia porque conllevaría la imposibilidad de distinguir entre la arbitrariedad y la discrecionalidad judicial.

\begin{abstract}
:
The living or evolving methodologies are widely used in interpretive practices of constitutional and international courts. Nevertheless, defences and criticisms of those methodologies hardly ever offer a systematic approach to interpretative stages of living constitutionalism. This article aims at remedying such insufficiency of constitutional theory through a systematic reconstruction of interpretative stages entailed by living constitutionalism. Then, I will identify the semantic presuppositions of living or evolving methodologies. The outcomes of this work will argue that those presuppositions lie on conventional semantics; that is, the extension of concepts refers more to social consensus than constitutional goods protected through rights. The former entails an impossibility to distinguish between arbitrariness and judicial discretion.
\end{abstract}

\title{
Palabras clave:
}

Constitucionalismo evolutivo; interpretación constitucional; derechos fundamentales; metodología; semántica convencionalista.

\section{Keywords:}

Living constitutionalism; constitutional interpretation; basic rights; methodology; conventional semantics. 